\title{
A series of technologies that advance the goal of data sharing
}

\author{
Ganghua Lin \\ National Astronomical Observatory, Chinese Academy of Sciences, \\ Datun Rd. 20A, Chaoyang District, Beijing, 100012, P. R. China, \\ email: lgh@bao.ac.cn
}

\begin{abstract}
One of the purposes of a Virtual Observatory is to facilitate data sharing. Data products from the Solar Multi-Channel Telescope and the Solar Radio Telescope at Huairou Solar Observing Station, Beijing, are not only used for solar research but also for solar activity and space environment predictions. To provide these services, we have exploited a number of technologies, which we discuss in this article. These include the setting up of a WWW server, a local area network, network security facility, data processing software, etc. We discuss the implementation of a Virtual Solar Observatory (VSO) and show how it meets various user requirements for unified international metadata. We also discuss future plans for further development of the system.
\end{abstract}

Keywords. Virtual solar observatories, astronomical data processing, solar physics

\section{The virtual solar observatory concept}

The Solar Multi-Channel Telescope at Huairou Solar Observation Station, Beijing comprises a local magnetic field, full-disc vector magnetograph, a full-disc $\mathrm{H}$-alpha telescope, etc. The resulting data are not only used for scientific research but also for solar activity predictions and space environment predictions. Direct users of the data products include solar physicists, the Solar Activity Prediction Centre (SAPC) of the Chinese Academy Sciences (CAS), the Space Environment Prediction Centre (SEPC) of CAS, university students and the public. To provide these services requires real-time or quasi real-time data, databases and database management software. We are therefore exploiting a number of technologies to provide these services.

\section{ICT technologies in the VSO}

\subsection{Setting up $W W W$ servers}

Our web page is a shop window for the VSO. Our website provides an introduction to our telescopes, our data and metadata sets that can be downloaded, simple data processing programs, etc. We also publish a Solar News column so that more users can learn about and use the data on our site. To ensure easy access, our data are available in FITS format. We have also made available translation software to transform our older local data into FITS files. Every day, the latest data and vector magnetic field image are added to the web site. The rapidly increasing volume of data and the website software infrastructure are backed up regularly, thereby allowing us to recover promptly from failures to maximize the up-time of our website. 


\subsection{Building up the network}

The Huairou Solar Observation Station (HSOS) is located on a small island near the north bank of the Huairou Reservoir, $6.5 \mathrm{~km}$ west of the county seat of Huairou and 60 $\mathrm{km}$ north of Beijing. At the time the website was set up, there was no internet connection to the station, so the only solution was to transmit the data by telephone line to the WWW server at headquarters. We used a Linux platform for the server because of its relative immunity to viruses and because of its relative stability. We used the telephone connection with $64 \mathrm{kbps}$ modems at each end for about three years. Because of the need for transmitting data in real time, we started a project to build up a fibre-based Local Area Network (LAN) connecting the various domes and and buildings in the Huairou Solar Observing Station. The LAN has a transmitting speed of $100 \mathrm{Mbps}$, and can even go up to 1000 Mbps when necessary. The Wide Area Network was recently upgraded to $10 \mathrm{Mbps}$ and is enough to satisfy current needs.

\subsection{Network security}

Unfortunately the network not only brings us high-speed connections, but also viruses and the threat of hacker invasions and network attacks. In HSOS there are various telescopes, instruments, data processing and data transmitting systems, all of which need to be protected. The various safety measures employed are shown in Fig. 1. Firstly, a network virus server was installed to protect every computer in HSOS. The server controls the upgrading and scanning of the client ends. The second network security element is a firewall to prevent invasion from outside the network. The third element is the invasion detection system, which complements the firewall and warns the network administrator of abnormal information transfers. The last element is a leak scan system which can find leaks in a computer operating system and report them to the administrator for corrective action.

\subsection{Data processing software}

Users of the data products are divided into a few groups: people with programming experience who are able to process data; people who know about solar physics but know less about the data and data processing methods; and lastly beginners who have neither programming nor data analysis skills. There is much time spent and much duplicated effort by various users to write software for manipulating and processing data.

An integrated data processing system is clearly advantageous as it reduces duplication of effort and improves data processing efficiency. Beginners can start processing data with a simple mouse click and thus obtain the results they need without having to learn all the specialized programming and data manipulation skills previously required.

The software makes use of a Graphical User Interface (GUI) to allow the user to:

- read files in different formats (FITS or local), either as single files or in batch mode;

- convert from FITS to different image formats, such as PS, GIF and JPEG;

- write data in the several common image formats;

- make contour plots;

- calibrate data;

- making vector magnetic images;

- make a movie from image files and write it out in AVI or MPEG format;

- edit and annotate images with text and graphics of various colors, sizes and shapes.

During image processing users can adjust parameters dynamically until they obtain satisfactory results. Users can enter parameters such as position coordinates, magnetic field strength, tilt angles, etc. at any point. The software will be extended to include roll correction, stretching, translation, solar rotation compensation, image co-alignment, etc. 


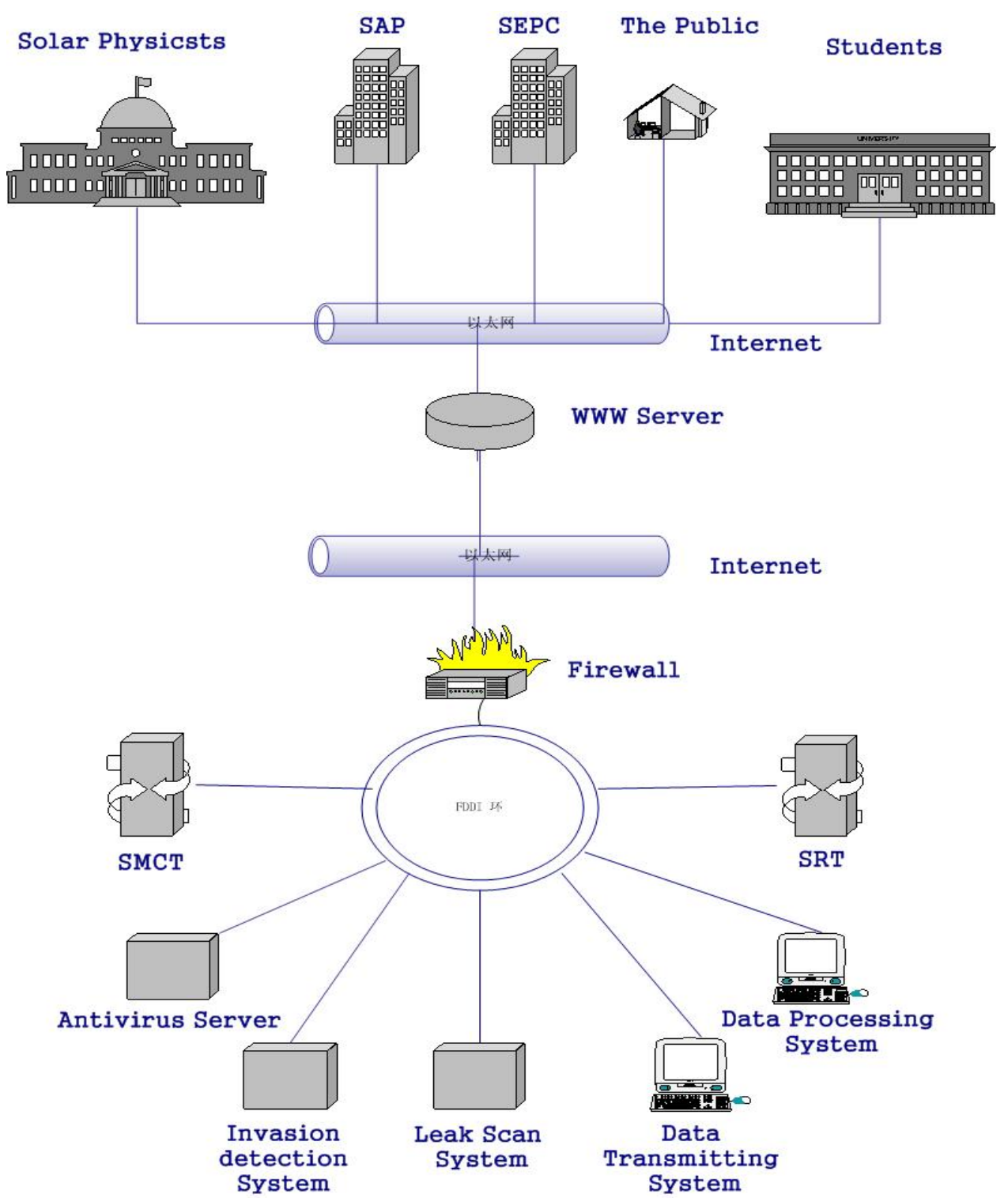

Figure 1. Schematic representation of the system and technologies used to supply data and services to various user groups.

We employ an object oriented approach to programming because of advances of object oriented programming in compiler technology. These advances make the software easy to maintain and adapt to other uses. For instance, with object-oriented programming, once a graph is drawn, all its attributes like color, size, text and markers can be modified interactively with a few mouse clicks. Three-dimensional graphs can be rotated with a mouse movement to be able to visualize the data from different view points. Several curves with different attributes can be superimposed in one graph. In this way a foundation for developing further functionality is established. 


\section{Further effort}

\subsection{Uploading data in real time}

Having the assurance of secure network access, we have the ability to transmit data in real time and at a high speed. We are able to respond to requests of users for data promptly and automatically.

\subsection{Automatic updates of web pages}

This function helps users learn what is happening on the surface of Sun in near real time.

\subsection{Developing query tools}

When searching through data sets, the availability of query tools greatly enhances efficiency. Examples of this would be querying data from solar active regions at low and high latitudes, or studying rotation-related phenomena. Hence designing good query tools is one of our focus areas. Connecting databases with the data processing software is another direction of our efforts.

\subsection{Developing virtual solar observatories (VSO)}

The VSO is a new kind of research tool for solar physics that will enable progress in many significant solar research problems. Data from new observing instruments will be published online in the VSO. The availability of our data in the VSO is likely to produce new research projects. We will link the VSO web site to our web site so that more users can learn about it and use it.

\section{Acknowledgements}

I would like to acknowledge support and help provided by my colleagues and by the employees of HSOS during the work described in this paper. 